\title{
INFECTIVE ENDOCARDITIS DUE T O SALMONELLA TYPHI
}

\author{
Khanal B, Sharma S K*, Deb M*
}

\section{ABSTRACT}

Rheumatic Heart Disease (RHD) remains an important predisposing lesion for infective endocarditis (IE) in developing countries. IE due to Salmonella typhi is rare but associated with potentially morbid complications.We report a case of Salmonella typhi in a patient with rheumatic heart disease. The diagnosis of IE was confirmed on the basis of Duke's clinical criteria. She responded to ciprofloxacin and gentamicin.

\section{Key Words: Rheumatic Heart Disease, Infective endocarditis, Salmonella typhi.}

\section{INTRODUCTION}

Infective endocarditis (IE) remains an important cause of morbidity and mortality The range of microbial species that cause IE is extraordinarily wide and varies form centre to centre. However, a few species account for majority of infection. Gram positive cocci have always dominated the scene as major etiologic agents. Gram negative bacilli (GNB) other than Hemophilus, Actinobacillus, Cardiobacterium, Eikenella and Kingella (HACEK) are regarded as less frequent cause of endocarditis. They are associated with certain percentage of Endocartitis in Intravenous drug abuser (IVDU)and Prosthetic valve endocarditis (PVE). ${ }^{1}$ We report a case of Salmonella typhi endocarditis in a patient with rheumatic heart disease.

\section{CASE REPORT}

A 27 years housewife, who was a diagnosed case of Rheumatic heart disease (RHD) with mitral stenosis (MS) and mitral regurgitation (MR) presented to the emergency of BP Koirala Institute of Health Sciences Hospital (BPKIHS), Dharan, Nepal, with 15 days history of fever, shortness of breath and swelling of the whole body. She was on regular prophylaxis for rheumatic fever with long-acting penicillin. There was no history of dental extraction, surgical procedure and abortion. She was evaluated one month prior to admission for complaints of breathlessness. Echocardiography done at that time showed moderate MS, moderate MR and mild tricuspid regurgitation.

Clinical examination revealed enlarged spleen,which was palpable $4 \mathrm{~cm}$ below the costal margin, firm and non-tender. Chest and neurological examination was unremarkable. Laboratory investigations revealed hemoglobin level of 10 gram \% Erythrocytic sedimentation rate (ESR), blood sugar, renal profiles were within normal limits. Urine examination showed plenty of Red Blood Cells. Pus cells and casts were absent. Three blood samples were drawn for culture. Salmonella typhi, identified by standard microbiological method and agglutination with group and type specific antisera was isolated from all three samples. ${ }^{2}$ The organism was sensitive to amikacin, chloramphenicol, ciprofloxacin and gentamicin and resistant to ampicillin and cotrimoxazole. Phage typing and Biotyping were carried out in National Salmonella Phage typing centre, Lady Hardinge Medical College, New Delhi. This isolate belonged to phage type A and Bio type I.

* B.P. Koirala Institute of Health Sciences, Dharan, Nepal.

** Vardhman Mahavir Medical College and Safdarjung Hospital, New Delhi

Address for correspondence : Dr. Basudha Khanal

B.P. Koirala Institute of Health Sciences, Dharan, Nepal.

Email: basudhak@yahoo.com 
Transthoracic echocardiography (TTE) done at this point revealed large vegetation attached to mitral leaflets in addition to her valvular defects. On the basis of Dukes clinical criteria diagnosis of IE was confirmed ${ }^{3}$. Patient was treated with ciprofloxacin and gentamicin to which she responded favourably.

\section{DISCUSSION}

Any bacteria can cause infective endocarditis but Gram positive cocci account for the majority of cases. Although most of the species of Gram negative bacilli (GNB) that colonize and /or infect human have been reported to cause bacteremias, they are associated with a small proportion of cases of native valve endocarditis .HACEK, a nutritionally fastidious group of GNB, forms a significant subgroup associated with IE. However, cases of other common aerobic GNB endocarditis are notably rare, though these species frequently cause Gram negative sepsis. ${ }^{1,4}$ Low adhesiveness of gram negative enteric bacilli to heart valve and fibrin and susceptibility of many strains to complement mediated bacteriolysis, probably are the causes of this disparity. ${ }^{1,2}$ Despite these factors two special populations, IVDU and patients with prosthetic valves are at increased risk of GNB endocarditis.

Cases of S. typhi endocarditis though rare, are reported in literature. ${ }^{1,45}$ Majority of patients with salmonella endocarditis have pre-existing disease of mitral and aortic valve. This patient had RHD involving mitral and tricuspid valves. Reports from western countries suggest that endocardial infection with salmonella is predominantly a disease of the elderly. Finding in India differs from this report where young age group with native valve defects are involved. ${ }^{6}$ This patient had the predisposing factor same as that seen in common cases of IE in India.

The crucial information in a case of Salmonella endocarditis is to distinguish it from the case of enteric fever occurring in a patient with damaged heart valve. The recent development of vegetation in this patient as proven by TTE performed after 30 days interval and growth of S. typhi from three blood cultures fulfills two major Dukes clinical diagnostic criteria for IE. Moreover three minor criteria (predisposing heart lesion, fever and microscopic hematuria) were also present.

Although the overall incidence of Salmonella endocarditis is low $(0.2-0.8 \%)$, it is known for higher rate of complications, relapse and mortality in absence of appropriate and timely treatment. Effective management of patient with IE therefore rests on the isolation and identification of the etiologic agent and institution of appropriate antibiotics.

This patient was receiving regular prophylaxis with long acting penicillin for rheumatic fever/RHD . She developed IE with S. typhi, which shows inherent resistance to penicillin. It is therefore concluded that any patient presenting with symptoms of IE should be bacteriologically evaluated before starting the empirical therapy.

\section{ACKNOWLEDGEMENT}

The authors thank Dr. Geeta Mehta, National Salmonella Phage Typing Centre, Lady Hardinge Medical College, New Delhi for phage typing and bio typing the strain.

\section{REFERENCES}

1. Durack DT. Infective Endocarditis In: Alexander RW, Schlant RC, Fuster V, Eds. The Heart, $9^{\text {th }}$ edn. New York: MoGraw Hill, 1998: 2205-2239.

2 Forbes BA, Sahm DF, Weissfeld AS, eds. Bailey and Scott's Diagnostic Microbiology. St.Louis: Mosby; 1998.

3. Durack DT, Lukes AS, Bright DK, et al. New criteria for diagnisis of Infective Endbcarditis: utilization of specific echocardiographic findings. Am J Med. 1994; 96: 200-209.

4. Geraci JE, Wilson WR, Endocarditis due to Gram negative bacteria. Report of 56 cases. Mayo Clin Proc 1982; 57: 14548.

5. Du Plessis JP, Govendrageloo K, Levin SE. Right sided endocaritis due to Salmonelal typhi . Pediatric Cardiology 1997; 18: 443-44.

6. Ghadage DP, Bal AM. Infective endocarditis due to an unusual serotype of Salmonella. Indian Heart J 2001; 53: 350-51. 